\title{
PERMUTING TRIDERIVATIONS OF PRIME AND SEMIPRIME RINGS
}

\author{
HASRET YAZARLI
}

Received 28 April, 2015

\begin{abstract}
The purpose of this paper is to prove some results concerning permuting triderivations and permuting generalized triderivations on prime and semiprime rings which partially extend some results contained in [9] and [10].
\end{abstract}

2010 Mathematics Subject Classification: 16A12; 16A68; 12A72

Keywords: prime ring, semiprime ring, permuting triderivation, permuting generalized triderivation

\section{INTROdUCTION AND PRELIMINARIES}

Throughout this work, $R$ will represent an associative ring and $Z$ will denote the center of $R$. We shall write $[x, y]$ for $x y-y x$. Recall that a ring $R$ is prime, if $x R y=\{0\}$ implies $x=0$ or $y=0$, and it is semiprime if $x R x=\{0\}$ implies $x=0$.

It is very interesting and important that the similar properties of derivation which is the one of the basic theory in analysis and applied mathematics are also satisfied in the ring theory. The commutativity of prime rings with derivations was introduced by Posner in [12]. An additive map $d: R \rightarrow R$ is called derivation if $d(x y)=$ $d(x) y+x d(y)$ holds for all $x, y \in R$. Recently, a lot of work has been done on commutativity of prime rings with derivation (see [1], [2], [3], [14]).

In [4], Bresar defined concept of generalized derivation. An additive map $d$ : $R \rightarrow R$ is called generalized derivation if there exists a derivation $\alpha$ of $R$ such that $d(x y)=d(x) y+x \alpha(y)$ for all $x, y \in R$. Thus the concept of generalized derivation contains both the concepts of a derivation and of a left multiplier (i.e., additive maps satisfying $f(x y)=f(x) y$ for all $x, y \in R)$. Basic examples are derivations and generalized inner derivations (i.e., maps of type $x \mapsto a x+x b$ for some $a, b \in R$ ). In [4], Bresar showed that if $R$ has the property that $R x=\{0\}$ implies $x=0$ and $h: R \rightarrow R$ is any function, $d: R \rightarrow R$ is any additive map satisfying $d(x y)=$ $d(x) y+x h(y)$ for all $x, y \in R$, then $d$ is uniquely determined by $h$ and moreover $h$ must be derivation. In [5], Bresar defined concept of generalized bi-derivation and investigated some its properties. 
In [7] and [8], Maksa defined biderivation in ring theory mutually to partial derivations and examined some properties of this derivation. A map $D(.,):. R \times R \rightarrow R$ is said to be symmetric if $D(x, y)=D(y, x)$ for all $x, y \in R$. A map $d: R \rightarrow R$ defined by $d(x)=D(x, x)$ is called the trace of $D(.,$.$) where D(.,):. R \times R \rightarrow R$ is a symmetric map. It is clear that if $D(.,$.$) is biadditive (i.e., additive in all arguments),$ then the trace $d$ of $D(.,$.$) satisfies the identity d(x+y)=d(x)+d(y)+2 D(x, y)$ for all $x, y \in R$. A symmetric biadditive map $D(.,):. R \times R \rightarrow R$ is called symmetric biderivation if $D(x z, y)=D(x, y) z+x D(z, y)$ for all $x, y, z \in R$. For any $y \in R$, the map $x \mapsto D(x, y)$ is a derivation. The trace of $D(.,$.$) is an even function. In [11]$ and [13], the authors investigated some properties of symmetric biderivation.

In [10], Öztürk defined concept of permuting triderivation. A map $D(., .,$.$) :$ $R \times R \times R \rightarrow R$ is called permuting if $D(x, y, z)=D(x, z, y)=D(z, x, y)=$ $D(z, y, x)=D(y, z, x)=D(y, x, z)$ hold for all $x, y, z \in R$. A map $d: R \rightarrow R$ defined by $d(x)=D(x, x, x)$ is called trace of $D(., .,$.$) , where D(., .,):. R \times R \times$ $R \rightarrow R$ is a permuting map. It is obvious that, if $D(., .,):. R \times R \times R \rightarrow R$ is permuting triadditive (i.e., additive in both arguments ), then the trace of $D(., .,$.$) satisfies$ the relation $d(x+y)=d(x)+d(y)+3 D(x, x, y)+3 D(x, y, y)$ for all $x, y \in R$. A permuting triadditive map $D(., .,):. R \times R \times R \rightarrow R$ is called permuting triderivation if $D(x w, y, z)=D(x, y, z) w+x D(w, y, z)$ for all $x, y, z, w \in R$. The trace of $D(., .,$.$) is an odd function. Let D(., .,$.$) be a permuting triderivation of R$. In this case, for any fixed $a \in R$ and for all $x, y \in R$, a map $D_{1}(.,):. R \times R \rightarrow R$ defined by $D_{1}(x, y)=D(a, x, y)$ and a map $d_{2}: R \rightarrow R$ defined by $d_{2}(x)=D(a, a, x)$ are a symmetric biderivation (in this meaning, permuting 2-derivation is a symmetric biderivation) and a derivation, respectively. In [9] and [15], the authors investigated some properties of permuting triderivation.

Lemma 1 ([6, page 6, Corollary 2]). If $R$ is a semiprime ring and $I$ is an ideal of $R$, then $I \cap A n n I=\{0\}$.

Lemma 2 ([10, Lemma 5]). Let $R$ be a 2,3-torsion free ring, $D$ a permuting triadditive map of $R$ and $d$ the trace of $D$. If $d(x)=0$ for all $x \in R$, then $D=0$.

In this paper, our aim is to prove some results concerning permuting triderivations and permuting generalized triderivations on prime and semiprime rings which partially extend some results contained in [9] and [10].

\section{RESULTS}

Lemma 3. Let $R$ be a 2,3-torsion free prime ring and $\{0\} \neq I$ be an ideal of $R$. If $D$ is a permuting triderivation such that $D(x, x, x)=0$ for all $x \in I$, then either $D=0$ or $R$ is commutative.

Proof. Let $D(x, x, x)=0$ for all $x \in I$. Replacing $x$ by $x+y$ for all $x, y \in I$, we get

$$
D(x, x, y)+D(x, y, y)=0
$$


Replacing $y$ by $-y$ in 2.1 and comparing with 2.1, we have

$$
D(x, x, y)=0 \text {, for all } x, y \in I .
$$

Replacing $y$ by $r y, r \in R$, we get $D(x, x, r) y=0$. Replacing $x$ by $x+z, z \in I$, we get $D(x, z, r) y=0$. Substituting $s x$ for $x$, we obtain that $D(s, z, r) x y=0, s \in$ $R$. Substituting $t z$ for $z$, we get $D(s, t, r) z x y=0$. From here $D(s, t, r) I[x, y]=$ $\{0\}$. Primeness of $R$ yields $[x, y]=0$ or $D(s, t, r)=0$, for all $x, y \in I$ and $s, t, r \in R$. Thus we have $[I, I]=\{0\}$ and $R$ is commutative or $D=0$.

Theorem 1. Let $R$ be a 2,3-torsion free prime ring and $\{0\} \neq I$ be an ideal of $R$. If $D$ is a permuting triderivation with trace $d$ such that $D(d(x), x, x)=0$ for all $x \in I$, then either $D=0$ or $R$ is commutative ring.

Proof. For any $x, y \in I$,

$$
D(d(x+y), x+y, x+y)+D(d(-x+y),-x+y,-x+y)=0
$$

and since $R$ is 2,3-torsion free we get

$$
\begin{aligned}
0 & =2 D(d(x), x, y)+D(d(y), x, x)+6 D(D(x, y, y), x, y) \\
& +3 D(D(x, x, y), x, x)+3 D(D(x, x, y), y, y) .
\end{aligned}
$$

Replacing $y$ by $y+z$ in 2.2 and using 2.2, we obtain

$$
\begin{aligned}
0 & =D(D(y, z, z), x, x)+D(D(y, y, z), x, x)+4 D(D(x, y, z), x, y) \\
& +2 D(D(x, y, y), x, z)+4 D(D(x, y, z), x, z)+2 D(D(x, z, z), x, y) \\
& +2 D(D(x, y, y), x, z)+D(D(x, x, y), z, z)+D(D(x, x, z), y, y) \\
& +2 D(D(x, x, z), y, z)
\end{aligned}
$$

since $R$ is 3 -torsion free.

Replacing $y$ by $-y$ in 2.3 and comparing with 2.3 , we get

$$
\begin{aligned}
0 & =D(D(y, y, z), x, x)+4 D(D(x, y, z), x, y)+4 D(D(x, y, y), x, z) \\
& +D(D(x, x, z), y, y)
\end{aligned}
$$

since $R$ is 2-torsion free.

Replacing $z$ by $y z$ in 2.4 and using 2.4, we get

$$
\begin{aligned}
0 & =d(y) D(x, x, z)+D(x, x, y) D(y, y, z)+4 D(x, y, y) D(x, y, z) \\
& +4 D(x, x, y) D(x, y, z)+D(x, x, y) D(y, y, z)+d(y) D(x, x, z) .
\end{aligned}
$$

Replacing $y$ by $x$ in 2.5, we get

$$
d(x) D(x, x, z)=0, \text { for all } x, z \in I
$$

since $R$ is 2,3-torsion free.

Replacing $z$ by $y z, y \in I$ in 2.6 and using 2.6, we have

$$
d(x) y D(x, x, z)=0 \text {, for all } x, y, z \in I .
$$


Therefore $d(x) y d(x)=0$ for all $x, y \in I$. And so, $y d(x) R y d(x)=\{0\}$. Since $R$ is prime ring, we get $y d(x)=0$ for all $x, y \in I$. That is, $d(x)=0$ for all $x \in I$. From Lemma 3, we obtain that $D=0$ or $R$ is commutative ring.

Theorem 2. Let $R$ be a 2,3,5-torsion free prime ring and $I$ be an ideal of $R$. If $D_{1}, D_{2}$ are the permuting triderivations of $R$ with traces $d_{1}, d_{2}$ respectively, such that $d_{1}(x) d_{2}(x)=0$ for all $x \in I$, then either $D_{1}=0$ or $D_{2}=0$ unless $R$ is commutative.

Proof. Assume that $R$ is not commutative. Then linearization of the relation

$$
d_{1}(x) d_{2}(x)=0 \text { for all } x \in I
$$

give as

$$
\begin{aligned}
0 & =d_{1}(x) d_{2}(y)+3 d_{1}(x) D_{2}(x, x, y)+3 d_{1}(x) D_{2}(x, y, y) \\
& +d_{1}(y) d_{2}(x)+3 d_{1}(y) D_{2}(x, x, y)+3 d_{1}(y) D_{2}(x, y, y) \\
& +3 D_{1}(x, x, y) d_{2}(x)+3 D_{1}(x, x, y) d_{2}(y)+9 D_{1}(x, x, y) D_{2}(x, x, y) \\
& +9 D_{1}(x, x, y) D_{2}(x, y, y)+3 D_{1}(x, y, y) d_{2}(x)+3 D_{1}(x, y, y) d_{2}(y) \\
& +9 D_{1}(x, y, y) D_{2}(x, x, y)+9 D_{1}(x, y, y) D_{2}(x, y, y) .
\end{aligned}
$$

Replacing $y$ by $-y$ in 2.8 , and comparing with 2.8 , we have

$$
\begin{aligned}
0 & =d_{1}(x) D_{2}(x, y, y)+d_{1}(y) D_{2}(x, x, y)+D_{1}(x, x, y) d_{2}(y) \\
& +3 D_{1}(x, x, y) D_{2}(x, x, y)+D_{1}(x, y, y) d_{2}(x)+3 D_{1}(x, y, y) D_{2}(x, y, y),
\end{aligned}
$$

since $R$ is 2,3 -torsion free.

Replacing $y$ by $y+z, z \in I$ in 2.9 and using 2.9, we get

$$
\begin{aligned}
0 & =2 d_{1}(x) D_{2}(x, y, z)+d_{1}(y) D_{2}(x, x, z)+d_{1}(z) D_{2}(x, x, y) \\
& +3 D_{1}(y, y, z) D_{2}(x, x, y)+3 D_{1}(y, y, z) D_{2}(x, x, z)+3 D_{1}(y, z, z) D_{2}(x, x, y) \\
& +3 D_{1}(y, z, z) D_{2}(x, x, z)+D_{1}(x, x, y) d_{2}(z)+3 D_{1}(x, x, y) D_{2}(y, y, z) \\
& +3 D_{1}(x, x, y) D_{2}(y, z, z)+D_{1}(x, x, z) d_{2}(y)+3 D_{1}(x, x, z) D_{2}(y, y, z) \\
& +3 D_{1}(x, x, z) D_{2}(y, z, z)+3 D_{1}(x, x, y) D_{2}(x, x, z)+3 D_{1}(x, x, z) D_{2}(x, x, y) \\
& +2 D_{1}(x, y, z) d_{2}(x)+6 D_{1}(x, y, y) D_{2}(x, y, z)+3 D_{1}(x, y, y) D_{2}(x, z, z) \\
& +6 D_{1}(x, y, z) D_{2}(x, y, y)+12 D_{1}(x, y, z) D_{2}(x, y, z)+6 D_{1}(x, y, z) D_{2}(x, z, z) \\
& +3 D_{1}(x, z, z) D_{2}(x, y, y)+6 D_{1}(x, z, z) D_{2}(x, y, z) .
\end{aligned}
$$

Replacing $y$ by $-y$ in 2.10 and comparing with 2.10, we get

$$
\begin{aligned}
0 & =D_{1}(y, y, z) D_{2}(x, x, z)+D_{1}(y, z, z) D_{2}(x, x, y)+D_{1}(x, x, y) D_{2}(y, z, z) \\
& +D_{1}(x, x, z) D_{2}(y, y, z)+D_{1}(x, y, y) D_{2}(x, z, z)+4 D_{1}(x, y, z) D_{2}(x, y, z) \\
& +D_{1}(x, z, z) D_{2}(x, y, y)
\end{aligned}
$$

since $R$ is 2,3 -torsion free. 
Replacing $z$ by $z+w, w \in I$ in 2.11 and using 2.11, we get

$$
\begin{aligned}
0 & =D_{1}(y, y, z) D_{2}(x, x, w)+D_{1}(y, y, w) D_{2}(x, x, z)+2 D_{1}(y, z, w) D_{2}(x, x, y) \\
& +2 D_{1}(x, x, y) D_{2}(y, z, w)+D_{1}(x, x, w) D_{2}(y, y, z)+D_{1}(x, x, z) D_{2}(y, y, w) \\
& +2 D_{1}(x, y, y) D_{2}(x, z, w)+4 D_{1}(x, y, z) D_{2}(x, y, w)+4 D_{1}(x, y, w) D_{2}(x, y, z) \\
& +2 D_{1}(x, z, w) D_{2}(x, y, y) .
\end{aligned}
$$

Replacing $y, z$ by $x$ in 2.12 and using 2.12, we have

$$
0=d_{1}(x) D_{2}(x, x, w)+D_{1}(x, x, w) d_{2}(x),
$$

since $R$ is 2,5 -torsion free.

Replacing $w$ by $w t, t \in I$ in 2.13 , we have

$$
\begin{aligned}
0 & =d_{1}(x) D_{2}(x, x, w) t+d_{1}(x) w D_{2}(x, x, t) \\
& +D_{1}(x, x, w) t d_{2}(x)+w D_{1}(x, x, t) d_{2}(x) .
\end{aligned}
$$

Using 2.13 in 2.14 , we get

$$
0=D_{1}(x, x, w)\left[t, d_{2}(x)\right]+\left[d_{1}(x), w\right] D_{2}(x, x, t) .
$$

Replacing $w$ by $d_{1}(x) w$ in 2.15 and using 2.15 , we get

$$
D_{1}\left(x, x, d_{1}(x)\right) w\left[t, d_{2}(x)\right]=0 \text { for all } x, w, t \in I .
$$

This implies that $D_{1}\left(x, x, d_{1}(x)\right) R w\left[t, d_{2}(x)\right]=\{0\}$ for all $x, w, t \in I$. Primeness of $R$ yields that either $D_{1}\left(x, x, d_{1}(x)\right)=0$ or $w\left[t, d_{2}(x)\right]=0$ for all $x, w, t \in I$. If $D_{1}\left(x, x, d_{1}(x)\right)=0$ for all $x \in I$, then conclusion follows from Theorem 1. Now consider the case when $w\left[t, d_{2}(x)\right]=0$ for all $x, w, t \in I$. Primeness of $R$ yields that $\left[t, d_{2}(x)\right]=0$ for all $x, t \in I$. By linearizing we get $\left[t, D_{2}(x, x, y)\right]=0$ for all $x, y, t \in I$. Replacing $y$ by $y z$, we have $D_{2}(x, x, y)[t, z]+[t, y] D_{2}(x, x, z)=0$ for all $x, y, z, t \in I$. In particular, $[t, y] D_{2}(x, x, y)=0$ for all $x, y, t \in I$. This implies that $[t, y] w D_{2}(x, x, y)=0$ for all $x, y, t, w \in I$. Since $R$ is not commutative, we also have $[I, I] \neq\{0\}$. Hence primeness of $R$ yields that $D_{2}(x, x, y)=0$ for all $x, y \in I$. From Lemma 3, we get $D_{2}=0$.

Definition 1. Let $R$ be a ring and $D: R \times R \times R \rightarrow R$ be a triadditive map. A triadditive map $\Delta: R \times R \times R \rightarrow R$ is called generalized derivation of $R$ associated with $D$ if for every $x, y \in R$, the map $z \mapsto \Delta(x, y, z)$ is a generalized derivation of $R$ associated with $D$, for every $y, z \in R$, the map $x \mapsto \Delta(x, y, z)$ is a generalized derivation of $R$ associated with $D$ and for every $x, z \in R$, the map $y \mapsto \Delta(x, y, z)$ is a generalized derivation of $R$ associated with $D$, i. e. for all $x, y, z, w \in R$,

$$
\begin{aligned}
& \Delta(x w, y, z)=\Delta(x, y, z) w+x D(w, y, z) \\
& \Delta(x, y w, z)=\Delta(x, y, z) w+y D(x, w, z) \\
& \Delta(x, y, z w)=\Delta(x, y, z) w+z D(x, y, w) .
\end{aligned}
$$


Example 1. Let $R$ be a ring. If $D$ is any triderivation of $R$ and $\alpha: R \times R \times R \rightarrow R$ is a triadditive map such that $\alpha(x, y, z w)=\alpha(x, y, z) w, \alpha(x, y w, z)=\alpha(x, y, z) w$ and $\alpha(x w, y, z)=\alpha(x, y, z) w$ for all $x, y, z, w \in R$, then $D+\alpha$ is a generalized $D$ triderivation of $R$.

Lemma 4. Let $R$ be a semiprime ring. Let $D: R \times R \times R \rightarrow R$ and $\Delta: R \times R \times$ $R \rightarrow R$ be two triadditive maps. If $\Delta$ is a generalized triderivation associated with $D$, then $D$ is a triderivation.

Proof. We compute $\Delta((x y) z, w, t)$ in two different ways. Then we have

$$
\begin{aligned}
\Delta((x y) z, w, t) & =\Delta(x y, w, t) z+x y D(z, w, t) \\
& =\Delta(x, w, t) y z+x D(y, w, t) z+x y D(z, w, t)
\end{aligned}
$$

and

$$
\Delta(x(y z), w, t)=\Delta(x, w, t) y z+x D(y z, w, t)
$$

for all $x, y, z, w, t \in R$. Comparing relations we obtain

$$
x(D(y, w, t) z+y D(z, w, t)-D(y z, w, t))=0 .
$$

Since $R$ is semiprime, we get $D(y z, w, t)=D(y, w, t) z+y D(z, w, t)$ for all $y, z, w, t \in R$. Similarly we obtain $D(x, z w, t)=D(x, z, t) w+z D(x, w, t)$ and $D(x, z, w t)=D(x, z, w) t+w D(x, w, t)$ for all $y, z, w, t \in R$.

Theorem 3. Let $R$ be a 2,3-torsion free prime ring and $I$ be a nonzero ideal of $R$. If $\Delta$ is a permuting generalized triderivation associated with permuting triderivation $D$ of $R$ with trace $d$ such that $\Delta(d(x), x, x)=0$ for all $x \in I$, then the following hold:

(i) $D=0$,

(ii) $R$ is commutative,

(iii) $d$ is commuting on $I$.

Proof. Let

$$
\Delta(d(x), x, x)=0 \text {, for all } x \in I
$$

Replacing $x$ by $x+y, y \in I$ in 2.16 and using 2.16, we get

$$
\begin{aligned}
0 & =2 \Delta(d(x), x, y)+\Delta(d(x), y, y)+\Delta(d(y), x, x)+2 \Delta(d(y), x, y) \\
& +3 \Delta(D(x, x, y), x, x)+6 \Delta(D(x, x, y), x, y)+3 \Delta(D(x, x, y), y, y) \\
& +3 \Delta(D(x, y, y), x, x)+6 \Delta(D(x, y, y), x, y)+3 \Delta(D(x, y, y), y, y) .
\end{aligned}
$$

Replacing $y$ by $-y$ in 2.17 and comparing with 2.17 , we have

$$
\begin{aligned}
0 & =\Delta(d(x), y, y)+2 \Delta(d(y), x, y)+6 \Delta(D(x, x, y), x, y) \\
& +3 \Delta(D(x, y, y), x, x)+3 \Delta(D(x, y, y), y, y),
\end{aligned}
$$

since $R$ is 2 -torsion free. 
Replacing $y$ by $y+z, z \in I$ in 2.18 and using 2.18, we get

$$
\begin{aligned}
0 & =2 \Delta(d(x), y, z)+2 \Delta(d(y), x, z)+2 \Delta(d(z), x, y)+6 \Delta(D(y, y, z), x, y) \\
& +6 \Delta(D(y, y, z), x, z)+6 \Delta(D(y, z, z), x, y)+6 \Delta(D(y, z, z), x, z) \\
& +6 \Delta(D(x, x, y), x, z)+6 \Delta(D(x, x, z), x, y)+6 \Delta(D(x, y, z), x, x) \\
& +6 \Delta(D(x, y, y), y, z)+3 \Delta(D(x, y, y), z, z)+6 \Delta(D(x, y, z), y, y) \\
& +12 \Delta(D(x, y, z), y, z)+6 \Delta(D(x, y, z), z, z)+3 \Delta(D(x, z, z), y, y) \\
& +6 \Delta(D(x, z, z), y, z) .
\end{aligned}
$$

Replacing $y$ by $-y$ in 2.19 and comparing with 2.19 , we obtain

$$
\begin{aligned}
0 & =2 \Delta(D(y, y, z), x, z)+2 \Delta(D(y, z, z), x, y)+\Delta(D(x, y, y), z, z) \\
& +4 \Delta(D(x, y, z), y, z)+\Delta(D(x, z, z), y, y),
\end{aligned}
$$

since $R$ is 3-torsion free.

Replacing $z$ by $y$ in 2.20, we get

$$
\begin{aligned}
0 & =2 \Delta(d(y), x, y)+2 \Delta(d(y), x, y)+\Delta(D(x, y, y), y, y) \\
& +4 \Delta(D(x, y, y), y, y)+\Delta(D(x, y, y), y, y)
\end{aligned}
$$

and so,

$$
0=2 \Delta(d(y), x, y)+3 \Delta(D(x, y, y), y, y),
$$

since $R$ is 2-torsion free.

Replacing $x$ by $x z, z \in I$ in 2.21 and using 2.21, we obtain that

$$
\begin{aligned}
0 & =2 x D(d(y), z, y)+3 D(x, y, y) D(z, y, y) \\
& +3 \Delta(x, y, y) D(z, y, y)+3 x D(D(z, y, y), y, y) .
\end{aligned}
$$

Replacing $x$ by $u x, u \in I$ in 2.22 and using 2.22, we get

$$
\begin{gathered}
0=D(u, y, y) x D(z, y, y)+\Delta(u, y, y) x D(z, y, y) \\
+u D(x, y, y) D(z, y, y)-u \Delta(x, y, y) D(z, y, y)
\end{gathered}
$$

since $R$ is 3-torsion free.

Replacing $u$ by $x$ in 2.23, we have

$$
0=D\left(x^{2}, y, y\right) D(z, y, y)+[\Delta(x, y, y), x] D(z, y, y)
$$

Replacing $z$ by $z u$ in 2.24 and using 2.24, we get

$$
\left(D\left(x^{2}, y, y\right)+[\Delta(x, y, y), x]\right) z D(u, y, y)=0 \text { for all } x, y, z, u \in I .
$$

Since $R$ is prime, we get either $D\left(x^{2}, y, y\right)+[\Delta(x, y, y), x]=0$ or $D(u, y, y)=$ 0 . In the last case and Lemma 3, we have that either $R$ is commutative or $D=0$.

If $D\left(x^{2}, y, y\right)+[\Delta(x, y, y), x]=0$ for all $x, y \in I$, then replacing $y$ by $y+z$, $z \in I$, we obtain that

$$
0=D\left(x^{2}, y, y\right)+2 D\left(x^{2}, y, z\right)+D\left(x^{2}, z, z\right)+[\Delta(x, y, y), x]
$$




$$
+2[\Delta(x, y, z), x]+[\Delta(x, z, z), x] .
$$

This implies that $0=D\left(x^{2}, y, z\right)+[\Delta(x, y, z), x]$, since $R$ is 2-torsion free. Replacing $z$ by $z w, w \in I$, we get

$$
0=\Delta(x, y, z)[w, x]+[z, x] D(x, y, w) .
$$

In the last relation, substituting $x$ for $w, y$, we get

$$
[z, x] d(x)=0
$$

Replacing $z$ by $r z, r \in R$ in 2.25 and using 2.25 , we obtain that

$$
[r, x] z d(x)=0 \text {, for all } x, z \in I, r \in R
$$

Replacing $z$ by $z x$, we have

$$
[r, x] z x d(x)=0 \text {, for all } x, z \in I, r \in R
$$

Multiplying by $x$ on the right side in 2.26, we get

$$
[r, x] z d(x) x=0 \text {, for all } x, z \in I, r \in R
$$

Comparing 2.27 and 2.28, we obtain that

$$
[r, x] z[d(x), x]=0 \text {, for all } x, z \in I, r \in R
$$

Replacing $r$ by $d(x)$ in 2.29, we get $[d(x), x] z[d(x), x]=0$ for all $x, z \in I$. This implies thal $z[d(x), x] R z[d(x), x]=\{0\}$ for all $x, z \in I$. Since $R$ is prime, we get $z[d(x), x]=0$ for all $x, z \in I$. And so, $[d(x), x] \in A n n(I)$. This implies that $[d(x), x] \in I \cap \operatorname{Ann}(I)=\{0\}$ for all $x \in I$. Hence $d$ is commuting on $I$.

\section{REFERENCES}

[1] H. E. Bell and M. N. Daif, "On derivations and commutativity in prime rings," Acta Math. Hungar., vol. 66, no. 4, pp. 337-343, 1995, doi: 10.1007/BF01876049.

[2] H. E. Bell and W. S. Martindale, III, "Semiderivations and commutativity in prime rings," Canad. Math. Bull., vol. 31, no. 4, pp. 500-508, 1988, doi: 10.4153/CMB-1988-072-9.

[3] M. Bre` sar, “A note on derivations," Math. J. Okayama Univ., vol. 32, pp. 83-88, 1990.

[4] M. Bre sar, "On the distance of the composition of two derivations to the generalized derivations," Glasgow Math. J., vol. 33, no. 1, pp. 89-93, 1991, doi: 10.1017/S0017089500008077.

[5] M. Bre sar, "On generalized biderivations and related maps," J. Algebra, vol. 172, no. 3, pp. 764-786, 1995, doi: 10.1006/jabr.1995.1069.

[6] I. N. Herstein, Rings with involution. Chicago III USA: Chicago lectures in mathematics, University of Chicago press, 1976.

[7] G. Maksa, "A remark on symmetric biadditive functions having nonnegative diagonalization," Glas. Mat. Ser. III, vol. 15, no. 2, pp. 279-282, 1980.

[8] G. Maksa, "On the trace of symmetric bi-derivations," C. R. Math. Rep. Acad. Sci. Canada, vol. 9, no. 6, pp. 303-307, 1987.

[9] D. Özden, M. A. Öztürk, and Y. B. Jun, "Permuting tri-derivations in prime and semi-prime gamma rings," Kyungpook Math. J., vol. 46, no. 2, pp. 153-167, 2006.

[10] M. A. Öztürk, "Permuting tri-derivations in prime and semi-prime rings," East Asian Math. J., vol. 15 , no. 2, pp. 177-190, 1999. 
[11] M. A. Öztürk and M. Sapanc1, "Orthogonal symmetric bi-derivation on semi-prime gamma rings," Hacet. Bull. Nat. Sci. Eng. Ser. B, vol. 26, pp. 31-46, 1997.

[12] E. C. Posner, "Derivations in prime rings," Proc. Amer. Math. Soc., vol. 8, pp. 1093-1100, 1957, doi: $10.2307 / 2032686$.

[13] J. Vukman, "Symmetric bi-derivations on prime and semi-prime rings," Aequationes Math., vol. 38, no. 2, pp. 245-254, 1989, doi: 10.1007/BF01840009.

[14] J. Vukman, "Commuting and centralizing mappings in prime rings," Proc. Amer. Math. Soc., vol. 109, no. 7, pp. 47-52, 1990, doi: 10.2307/2048360.

[15] H. Yazarli, M. A. Oztürk, and Y. Bae Jun, "Tri-additive maps and permuting tri-derivations," Commun. Fac. Sci. Univ. Ank. Sér. Al Math. Stat., vol. 54, no. 1, pp. 1-8, 2005.

Author's address

Hasret Yazarli

Cumhuriyet University, Department of Mathematics, Faculty of Science, 58140 Sivas, Turkey

E-mail address: hyazarli@cumhuriyet.edu.tr 